Methods The cross-sectional, mixed methods design was employed. The study population was 785 health care workers and non-health workers working at University of Port-Harcourt Teaching Hospital (Nigeria). The purposive sampling was used for qualitative study while the stratified random sampling technique was utilised for the quantitative study. Qualitative data were collected from fifteen respondents while a total of 511 questionnaires were administered at the study site. The qualitative data was analysed using inductive thematic analysis. The quantitative data was analysed using structural equation modelling (SEM).

Results The qualitative study suggested that quality improvement was perceived as most useful in influencing all the tree sub-components of readiness. Training is perceived as most useful in building readiness while it is perceived to be moderately useful in influencing the sub-component of readiness. The OLS estimates indicates that QI/QA exert a positive and significant effect on motivation $(\beta=0.004, p<0.05)$ and general capacity score $(\beta=0.28 \mathrm{p}<0.05)$ while it inversely but significantly exerts influence on innovation specific capacity $(\beta=-0.21 \times 10-3, p<0.05)$. The $\mathrm{SEM} /$ pathway analysis shows the direct and indirect routes of interactions among predictors of readiness after adjusting for confounders. All the explanatory variables have significant effect on readiness except gender which was dropped from the final model.

Conclusion The strength of evidence of how an evidencebased system for innovation support can influence readiness was established. Though readiness is a rate-determining step in ensuring robust and effective implementation outcomes for epidemic containment, exploring innovation outcomes and their amplification through explicitly target readiness dynamics requires further investigation.

\section{PO 8355 HOST GENETIC POLYMORPHISMS AND ASYMPTOMATIC MALARIA IN SOUTHERN GHANA}

${ }^{1}$ Paulina Otu*, ${ }^{2}$ Aminata Colle Lo, ${ }^{2}$ Ben Gyan, ${ }^{1}$ Yaw Afrane, ${ }^{2}$ Linda Eva Amoah. ${ }^{1}$ University of Ghana, Legon, Accra, Ghana; ${ }^{2}$ Immunology Department, Noguchi Memorial Institute for Medical Research, College of Health Sciences, University of Ghana, Accra, Ghana

\subsection{6/bmjgh-2019-EDC.74}

Background Despite several interventions through malaria control programmes, asymptomatic malaria is a major barrier to control as asymptomatic individuals serve as reservoirs from which others are re-infected. The mechanism by which these individuals remain asymptomatic is not well understood. Much work has been done in relation to human genes and their association to severe, mild and uncomplicated malaria. However, there is limited knowledge regarding host genetic factors and asymptomatic malaria.

Method In this study, we investigated the association between host genetic polymorphisms of glucose-6-phosphate dehydrogenase gene (G6PD), mannose binding lectin (MBLG54A), tumor necrotic factor alpha (TNF-G308A) and nitric oxide synthase 2 (NOS2-G954C) and the outcome of asymptomatic P. falciparum malaria in 150 healthy individuals in southern Ghana.

Results We found a significant association between G6PDd and asymptomatic malaria with a prevalence of $9.6 \%$ $(\mathrm{p}=0.035$, by chi-square test). All the individuals who were heterozygous and hemizygote deficient (5.3\% and $4.3 \%)$ were found to be asymptomatic. Individuals homozygous (GG) for TNF (G308A) were found to be highly asymptomatic $(\mathrm{p}=0.019$, by chi-square test). Regarding MBL (G54A) and
NOS (G954C), no significant association was found between these markers and asymptomatic malaria.

Conclusion Upon reviewing our data with other data from published work, we conclude that both heterozygous and hemizygous individuals with G6PD A- and homozygous individuals (GG) of TNF (G308A) polymorphisms could be predisposed genetically to asymptomatic malaria.

\section{PO 8356 ANGIOGENIC AND ANGIOSTATIC FACTORS IN THE SALIVA OF MALARIA PATIENTS}

${ }^{1}$ Cecilia Lekpor*, ${ }^{1}$ Felix Botchway, ${ }^{2} J o n a t h a n$ Stiles, ${ }^{2}$ Wilson 0 Nana. ${ }^{1}$ Department of Patholgy, University of Ghana, Accra, Ghana; ${ }^{2}$ Morehouse School of Medicine, Atlanta (GA), USA

\subsection{6/bmjgh-2019-EDC.75}

Background Malaria mortality is associated with exaggerated host responses to inflammatory factors such as C-X-C motif chemokine 10 (CXCL10) and host biomarkers such as angiopoietin 1 (Ang-1) and angiopoietin 2 (Ang-2). The aim of this study was to determine saliva levels of CXCL10, Ang-1 and Ang-2 and compare with plasma levels regarding their potential as biomarkers of malaria, which may be useful for further development of highly efficient non-invasive malaria detection methods.

Methods Case control study involving 213 subjects (119 with and 94 without malaria) aged 1-16 years. Haematological determination was done using Haematology Analyser. Plasmodium Lactate Dehydrogenase/Histidine Rich Protein-2 (pLDH/ HRP-2) Antigen rapid diagnostic test (RDT) were performed. Plasma and saliva levels of CXCL10, Ang-1 and Ang-2 were measured using Elisa kit. Data was presented as mean \pm standard error or median and interquartile range (IQR). A p-value $<0.001$ was considered statistically significant. Results There was decreased plasma levels of Ang-1 and increased plasma levels of CXCL10 and Ang-2 in individuals with malaria compared to those without malaria (Ang-1, $\mathrm{p}<0.009$; Ang-2, $\mathrm{p}<0.001$; CXCL10 $\mathrm{p}<0.001)$. Biomarker levels in both plasma and saliva in subjects with malaria and without malaria were correlated and a significant relationship was found between Ang -2 and CXCL10 which could be used to predict malaria severity $(p=0.001$ for Ang- 2 and $\mathrm{p}<0.01$ for CXCL10). Low Ang-1 and high Ang-2 in both plasma and saliva were significantly associated with increased risk of malaria severity: Ang-1, 2741.04 (1785.85-3582.68), $\mathrm{p}<0.009 ; \quad$ Ang-2, $3508.82 \quad$ (2139.61-5091.633.9), $\mathrm{p}<0.001$ and Ang-1, 720.27 (439.82-1086.74); 16.98 (10.0833.26), ( $p<0.001$ for all). Finally, Ang-2 was informative when combined with CXCL10 to predict malaria severity.

Conclusion These results provide insight into the use of saliva for a non-invasive diagnostic method and demonstrate that Ang-2 combined with CXCL10 is a promising predictive biomarker of malaria severity.

\section{PO 8369 BURULI ULCER: PATTERN OF PRESENTATION IN A NIGERIAN HOSPITAL}

${ }^{1}$ Chukwuanugo Ogbuagu*, ${ }^{2}$ Ifeoma Enweani, ${ }^{3}$ Agu Maurice, ${ }^{1}$ Ekenechukwu Ogbuagu, ${ }^{1}$ Obiageli Emelumadu. ${ }^{1}$ Nnamdi Azikiwe University Teaching Hospital, Nnewi, Nigeria; ${ }^{2}$ Nnamdi Azikiwe University, Awka, Nigeria; ${ }^{3}$ Nnewi Diocesan Hospital, Nnewi, Nigeria

10.1136/bmjgh-2019-EDC.76 
Background Buruli ulcer is one of the neglected tropical diseases. It is a chronic, debilitating, necrotising disease of the skin and soft tissue caused by Mycobacterium ulcerans. Most times, the pattern of presentation is neglected by the infected because it is regarded as a disease of the poor who have little or no access to healthcare. Living in rural often inaccessible areas and suffering from a triad of ignorance, stigma and poverty, this poor population fails to present early to a hospital.

Methods A retrospective review of patients who accessed care at the infectious disease clinic of Nnewi Diocesan Hospital, Nnewi, Southeast Nigeria, between 1 January to 31 December 2017. To achieve a complete inference, the results of laboratory wound swab culture of all patients were collated and matched with the clinical presentation. All cultures were done by a trained scientist of the German Leprosy and TB Relief Association (GLRA).

Results Review of data showed a total of 10120 patients of which 6402 were outpatients and 3718 were inpatients; they were between 1 and 86 years of age. There were 60 cases of limb ulcers of which wound swab culture was done. Fifty-four (54) were diabetic foot ulcers while five (5) were venous ulcers. Acid-fast bacilli were detected with Ziehl-Neelsen staining in one specimen and confirmed by the reference center.

Conclusion Most of the Buruli ulcer patients are found incidentally following late presentation at hospitals with a questionable ulcer/wound with a high index of suspicion on clinical examination. If Buruli ulcer is to be eradicated, an intensive rural epidemiological identification programme must be implemented to isolate the infected. The vicious cycle of ignorance, stigma and poverty needs to be broken by massive awareness and education campaigns.

\section{PO 8372 CULTURE-FREE APPROACHES FOR THE DIAGNOSIS AND MANAGEMENT OF PATIENTS WITH RIFAMPICIN RESISTANT TUBERCULOSIS: THE DIAMA PROJECT}

\begin{abstract}
${ }^{1}$ Faridath Massou*, 'Dissou Affolabi, ${ }^{2}$ Corinne Merle, ${ }^{3}$ Gemeda Abebe, ${ }^{4}$ Oumou Bah Sow, ${ }^{5}$ Bassirou Diarra, ${ }^{6}$ Osman El Tayeb, ${ }^{7}$ Ayou Gaye Diallo, ${ }^{8}$ Michel Kaswa, ${ }^{9}$ Jean Claude Ngabonziza Semuto, ${ }^{10}$ Melissa Sander, ${ }^{11}$ Philip Supply, ${ }^{12}$ Bouke De Jong. ${ }^{1}$ Supranational Reference Laboratory of Mycobacteria, Cotonou, Benin; ${ }^{2}$ Special Programme for Research and Training in Tropical Diseases, World Health Organization (WHO/TDR), Geneva, Switzerland; ${ }^{3}$ Jimma University, Jimma, Ethiopia; ${ }^{4}$ Service de Pneumophtisiologie, Guinea, Conakry; ${ }^{5}$ Université des Sciences, des Techniques et des Technologies de Bamako, SEREFO, Mali, Bamako; ${ }^{6}$ Damian Foundation, Ibadan, Nigeria; ${ }^{7}$ Université Cheikh Anta Diop, Senegal, Dakar; ${ }^{8}$ Institut National de Recherche Biomédicale (INRB), Kinshasa, DRC; ${ }^{9}$ Rwanda Biomedical Center (RBC), Kigali, Rwanda; ${ }^{10}$ The Tuberculosis Reference Laboratory Bamenda, Cameroon; ${ }^{11}$ Genoscreen, France, Lille; ${ }^{12}$ Institute of Tropical Medicine (ITM), Belgium, Antwerp
\end{abstract}

\subsection{6/bmjgh-2019-EDC.77}

Background Recent advances in molecular diagnostics, especially the Xpert MTB/Rif test, have reduced the time to diagnose rifampicin resistant tuberculosis (RR-TB). However, with this test only rifampicin resistance is diagnosed, leading to presumptive diagnosis of resistance to isoniazid and maybe other drugs. In addition, culture on monthly sputum samples is currently recommended by the World Health Organization (WHO) for follow-up of RR-TB patients under treatment. Unfortunately, culture is often not locally available, and samples need to be shipped from field to culture laboratories. The associated transport delays lead to high rates of contamination and false-negative culture, particularly in laboratories in low-resource settings. Many gaps for the diagnosis and management of RR-TB patients still need to be addressed and the DIAMA project (Diagnostics for multidrug-resistant tuberculosis in Africa) aims to address some of them.

Methods The TB Supranational Reference Laboratory of Benin leads a consortium of 11 partners involved in multiple-drug resistant TB care in Africa. The DIAMA project will explore the feasibility and accuracy of: i) diagnosing TB resistance to first and second line drugs through novel molecular multiplex assays developed by the company Genoscreen; ii) setting-up alternative culture-free approaches for the monitoring of patients' response to rifamipcin-resistant treatment; iii) piloting whether the implementation of software by Data2Care Technologies for real-time monitoring of molecular test results can reduce delays between diagnosis and treatment of RR-TB patients. This project is funded by EDCTP for a period of five years.

Conclusion Together, these advances could dramatically improve the currently dismal prognosis of multiple-drug resistant $\mathrm{TB}$ in health systems in resource-poor settings. Through this presentation, we will share the background information, the design of this project and its progress.

\section{PO 8381 REGULATING CLINICAL TRIALS DURING AN EBOLA EMERGENCY: THE LIBERIAN EXPERIENCE}

${ }^{1}$ Theophilus Ndorbor*, 'David Sumo, 'Juwe D Kercula, ${ }^{2}$ Ezekiel F Hallie. 'Liberia Medicines and Health Products Regulatory Aurthority, Monrovia, Liberia; ${ }^{2}$ School of Pharmacy, University of Liberia, Monrovia, Liberia

\subsection{6/bmjgh-2019-EDC.78}

Background Effective clinical trials oversight is a major function of a fully functional national medical products regulatory system. However, exercising clinical trial oversight in a resourcelimited environment is challenging, in particular during an Ebola outbreak or health emergency. Until the devastating Ebola virus disease (EVD) outbreak in 2014, the Liberia Medicines and Health Products Regulatory Authority (LMHRA) had no capacity for effective clinical trial regulation. This presentation describes the main challenges encountered by LMHRA in regulating clinical trials in Liberia during the largest EVD outbreak that affected West Africa in 2014 and 2015.

Methods By carefully documenting activities during the EVD outbreak, interviewing key stakeholders, and discussions among the LMHRA clinical trial committee, key challenges observed during the outbreak were identified and documented.

Results Limited financial resources, lack of expertise in clinical trials, inaccurate and insufficient information about the functions of the LMHRA, poor coordination among key stakeholders, and the lack of a well-developed regulatory framework, adversely influenced the LMHRA clinical trial oversight performance during the EVD outbreak.

Conclusion It is true that several challenges need to be addressed when regulating a clinical trial in a limited-resource environment during any disease outbreak or international medical emergency. However, the importance of building local expertise in clinical trials through mentorship and training cannot be overemphasised. By taking advantage of grants from developmental partners, national medicines regulatory authorities in resource-limited environments can develop capacity for clinical research oversight. 